\title{
Study protocol of sleep disorders in cancer patients of a reference hospital in Minas Gerais - cross-sectional observational study
}

\author{
Luísa Teixeira Pasqualotto1, Walquíria da Mata Santos², Cíntia Aparecida Santos', Sarah Cristina Vieira³, \\ Layra Fernanda Martins², Jacqueline Alves ${ }^{4}$, Rubia Lima Brandão ${ }^{4}$, Elisa Faccion Cruz Fideles ${ }^{4}$, \\ Camila Mariângela Pacheco ${ }^{5}$, Carolina Silva Dâmaso ${ }^{6}$, Newton Santos de Faria Júnior ${ }^{7}$
}

\begin{abstract}
Background: Sleep disorders are increasingly recognized as an important cause of morbidity and mortality. The poor quality of sleep is among the most frequent problems which patients with cancer have during treatment. Objectives: To verify the prevalence of sleep disorders in cancer patients attended at a reference hospital in the Center-West of Minas Gerais, Brazil. Methods: This research will be a cross-sectional observational study carried out by teachers and students from University of the State of Minas Gerais - UEMG, Divinópolis Unit, with subjects recruited from a reference hospital in the Center-West of Minas Gerais from March 2018 and February 2019. These patients will be evaluated clinically and will respond to Pittsburgh Sleep Quality Index (PSQI), Insonnia Severity Index (ISI), Epworth Sleepiness Scale (ESS), Berlin Questionnaire, European Organization for Research and Treatment of Cancer core Quality of life Questionnaire (EORTHC QLQ-30) and Beck Anxiety and Depression Inventory, respectively, assessing sleep quality, presence of sleep disorders, severity and presence of insomnia, excessive daytime sleepiness, risk for obstructive sleep apnea, quality of life and levels of anxiety and depression. Discussion: Currently, subjective instruments are used to evaluate the sleep pattern in cancer patients, since there is little information investigating the sleep disorders in these subjects. Recognizing these changes is fundamental as these symptoms can negatively affect the quality of life and their daytime performance.
\end{abstract}

Keywords: Sleep Disorders; Sleep; Cancer; Quality of Life.

\section{INTRODUCTION}

Sleep is defined as a restorative and healthy state, compared to rest and inactivity, and it is necessary to recover physical exhaustion due to the constant state of alertness and energy expenditure ${ }^{(1)}$. It can be considered as a complex biological process that alternates with the periods of wakefulness ${ }^{(2)}$. Additionally, it has been seen that sleep brings benefits that lead to the essential restorative state and to the proper functioning of the organism as maintenance of physical and mental health, which can modify the body temperature, cardiac work and hormonal production ${ }^{(3)}$. In 1997 the International Classification of Sleep Disorders was created by the American Association Sleep Medicine (AASM), in association with other companies, and it was subsequently reformulated ${ }^{(4)}$. This classification was necessary to discriminate and facilitate the understanding of the symptoms, etiology, pathophysiology and treatment. Subsequently, it was published in 2014, the International Classification of Sleep Disorders Third Edition (ICSD-3), which includes extensive literature review to list sleep disorders in detail, as well as the criteria used for diagnosis, being divided into 7 categories: insomnia, sleep related breathing disorders, central disorders of hypersomnolence, circadian rhythm sleep-wake disorders, parasomnias, sleep related movement disorders, other sleep disorders ${ }^{(5)}$.

Sleep-related breathing disorders are subdivided into obstructive sleep apnea (OSA), central sleep apnea, sleep-related hypoventilation, and sleep-related hypoxemia disorders. OSA is a highly prevalent disorder characterized by repetitive obstructions of the upper airways leading to intermittent hypoxia and sleep fragmentation ${ }^{(6)}$. These events are often associated with the reduction of oxyhemoglobin saturation and may occur excessive daytime sleepiness (EDS), verified by the Epworth Sleepiness Scale (ESS), or insomnia ${ }^{(7)}$. Sleep disorders have a prevalence of $10 \%$ to $48 \%$ and have been associated with chronic diseases such as systemic arterial hypertension, obesity, dyslipidemia, insulin resistance, diabetes mellitus, chronic pain, osteoporosis and

*Corresponding author: Newton Santos de Faria Júnior: PT, PhD. Address: Av. Paraná, 3001, Jardim Belvedere. Zip Code 35.501-170. University of the State of Minas Gerais - UEMG, Divinópolis Unit, Divinópolis, (MG), Brazil. E-mail: nsdfj@yahoo.com.br

${ }^{7}$ Professor, Physiotherapy Course of University of the State of Minas Gerais -Divinópolis Unit (UEMG), Divinópolis, Minas Gerais, Brazil.

Full list of author information is available at the end of the article.

Financial support: The authors declare that there was no financial support.

Submission date 13 October; Acceptance date 22 November 2018; Publication date 07 December 2018 
depression, considered as a major public health problem ${ }^{(3)}$. As for the diagnostic methods, the gold standard for sleep is the full-night polysomnography (PSG). This refers to the simultaneous recording of some physiological variables during sleep: stage and continuity of sleep, respiratory effort, oxyhemoglobin saturation, body position, electrocardiogram, body movements, electroencephalogram, electrooculogram, electromyography, airflow and body temperature ${ }^{(8)}$. However, to carry out this examination, a place with adequate physical structure and human resources with specific training is required, thus requiring a high financial investment and restricting their availability to some centers of the country. Thus, subjective instruments have been used both in clinical routine and in research protocols in the world scientific community, such as the Pittsburgh Sleep Quality Index (PSQI), a combination of quantitative and qualitative information about sleep.

The sleep regulatory mechanism in cancer patients is altered, being interrupted for a variety of reasons, as insomnia, nightmares, daytime sleepiness, nocturnal awakenings, difficulty returning to sleep and waking up very early ${ }^{(9)}$. According to the National Cancer Institute José Alencar Gomes da Silva (INCA) in Brazil, it is estimated that 600,000 new cases of cancer occurred in 2018-2019. The division of incidence by geographic region shows that the South and Southeast regions had $70 \%$ of the occurrence of cases, almost half of which are found in the Southeast region of the country ${ }^{(10)}$.

Sleep disorders in cancer patients can occur at different times in the course of the disease (diagnosis, treatment and terminal phase), resulting in decreased quality of life (QOL), impairment of mood and reduction of energy. In addition, the likelihood of these patients developing anxiety, depression and stress are high, and may persist for several months or even years after treatment, becoming chronic ${ }^{(11,12)}$. Some studies with these patients have shown that the prevalence of these sleep disorders are around $30 \%$ to $50 \%$. The main complaint is the difficulty to sleep and waking up several times at night, both at home and in the hospital, mainly related to pain. There are reports that 23 to $44 \%$ present these symptoms after two to five years of cancer treatment ${ }^{(12,13)}$. Although sleep disturbances in cancer patients are a major issue, information on this specific population is still limited when compared to its impacts on the general population ${ }^{(14)}$. Thus the objective of this study will be to verify the prevalence of sleep disorders in cancer patients attended at a reference hospital in Minas Gerais.

\section{METHODS}

\section{STUDY DESIGN AND ETHICAL CONSIDERATION}

This research will be a cross-sectional observational study carried out by teachers and students from University of the State of Minas Gerais - UEMG, Divinópolis Unit, with subjects recruited from a reference hospital in the Center-West of Minas Gerais from March 2018 and February 2019. The design of the study will follow the "Strengthening the Reporting of Observational Studies in Epidemiology (STROBE) statement" (Figure 1) ${ }^{(15)}$ and will agree to the ethical standards set in the Declaration of Helsinki, 1961 (review in Hong Kong, 1989, and Edinburgh, in Scotland, 2000) and the Regulatory Guidelines and Norms for research involving human beings from the National Health Council of the Brazilian Ministry of Health, resolution 196/96 updated by 466/2012. The present study was approved by the Ethics Committee in Research with Human Beings of the UEMG, protocol 2.418.202/2017. All the patients involved will sign the Statement of Consent Form, being allowed to leave at any time without charge.

\section{SUBJECTS}

The subjects participating in this study will be the ones assisted at a reference hospital in the Center-West of Minas Gerais, of both genders, over 18 years old, after agreeing to participate in the study and signing the Statement of Consent Form, without cognitive deficit that hinders comprehension in order to answer the questionnaires. Those who do not agree to participate in the study and/or have comorbidities that may influence the evaluation, diagnosis and/or prognosis at the outcome of the disease will be excluded. This sample

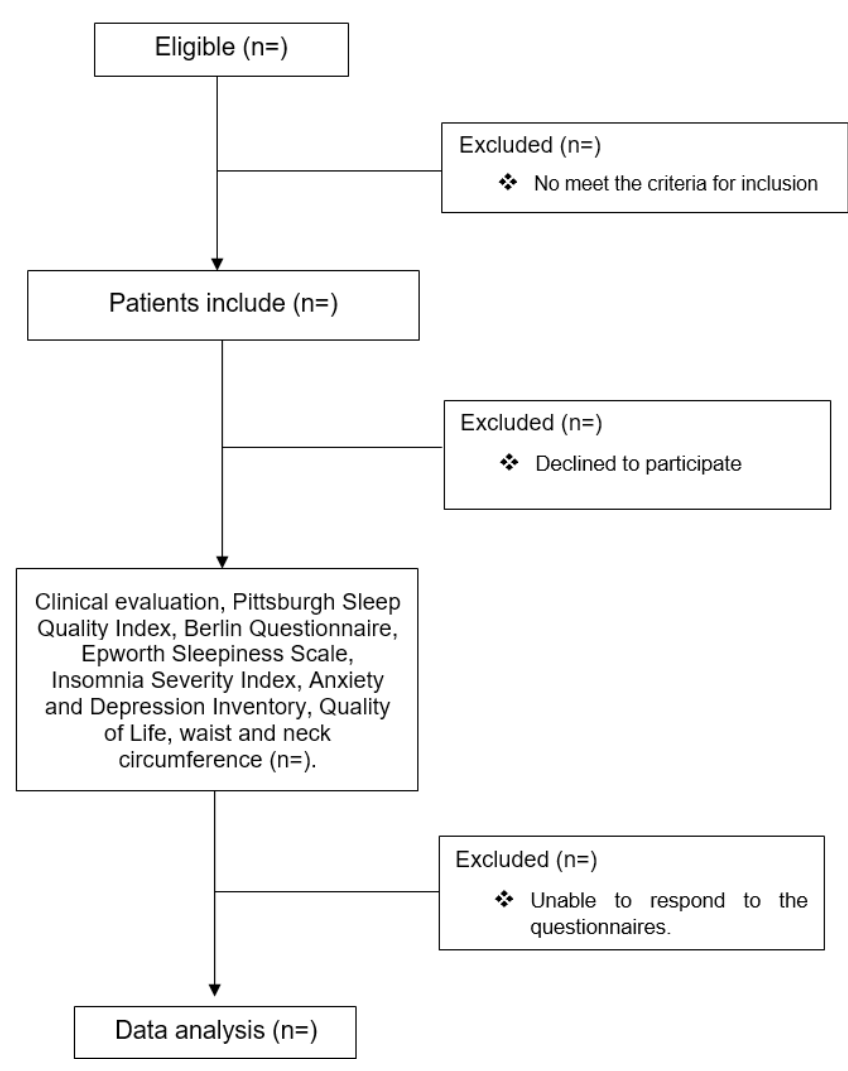

Figure 1. Flowchart of the study. 
will be consecutive and of convenience and later stratified as to the socio-demographic variables, comorbidities, type and location of cancer, whether or not in chemotherapeutic treatment, presence or not of sleep disorders and other variables pertinent to the study.

\section{PROTOCOL}

\section{CLINICAL}

The evaluation of the patients will be performed at a reference hospital in the Center-West of Minas Gerais, where personal data, objective evaluation of heart rate, respiratory rate, systemic arterial pressure, weight and height will be collected. The neck and waist circumference will be measured using a non-elastic tape with $1 \mathrm{~mm}$. With the subject in sitting position, the neck circumference will be measured on the anterior border of the cricoid cartilage and the waist circumference on the midpoint between the lower margin of the last rib and the iliac crest ${ }^{(16)}$. The evaluation of weight and height will be performed through an electronic scale (model 200/5, Welmy Indústria e Comércio Ltda, São Paulo, Brazil). The BMI calculation will be performed through the BMI classification of the World Health Organization. Whereas, systemic blood pressure will be measured by the auscultatory method after the subject is resting for 10 minutes $^{(17)}$.

\section{PITTSBURGH SLEEP QUALITY INDEX (PSQI)}

To evaluate the sleep quality of these patients, after clinical evaluation, will be applied the Portuguese version of the PSQI ${ }^{(18,19)}$, composed by 19 questions, divided into 7 parts scored separately. The sum of these points (interval: 0-21) provides an overall measure of sleep quality, with high scores indicating insufficient sleep ( $>5$ indicates poor sleep quality). The domains evaluated are related to sleep duration, subjective sleep quality, sleep latency, sleep disturbances, use of sleep-related medications, habitual sleep efficiency, and sleep impact on daytime dysfunctions.

\section{INSONNIA SEVERITY INDEX (ISI)}

In addition to the previous questionnaire will be applied the ISI, which evaluates the subjective symptoms and consequences of insomnia in daily activities, as well as the concerns. It is a self-administered questionnaire that quantifies the patient's perception about insomnia, consisting of seven items that investigate sleep initiation and maintenance, satisfaction with sleep patterns, interferences in daily activities, and degree of concern attributed to problems with sleep. Each item is assigned a value on a scale of 0 to 4 and the total score ranges from 0 to 28 , in which a score of 0 to 7 indicates absence of insomnia; 8 to 14 mild insomnia; 15 to 21 moderate insomnia; and 22 to 28 severe insomnia ${ }^{(20)}$. The Portuguese version of the ISI was valid and adequate to assess and evaluate such impacts in different contexts ${ }^{(21)}$ and the authors authorized its use.

\section{EPWORTH SLEEPINESS SCALE AND BERLIN QUESTIONNAIRE}

After ISI, two specific sleep questionnaires will be applied. To determine the risk for OSA, an individualized clinical questionnaire, called the Berlin Questionnaire will be used ${ }^{(22)}$. This questionnaire contains 10 items organized into three categories as follows: apnea and snoring, daytime somnolence and systemic arterial hypertension and obesity. All positively marked responses are considered risk factors for OSA. Patients will be classified as high risk or low risk for OSA. A patient is considered at high risk for OSA if two or more of the three categories are positive. As to determine the presence of EDS, will be applied the Portuguese version of ESS ${ }^{(23)}$. This is a simple and self-administered questionnaire with situations involving the occurrence of daytime sleepiness during normal daily activities in adults. Subjects will be instructed to rate their likelihood of feeling the urge to sleep or nap in eight situations, on a scale of 0 to 3 .

\section{BECK ANXIETY AND DEPRESSION INVENTORY}

Beck Anxiety Inventory (BAI) and Beck Depression Inventory (BDI) are questionnaires that evaluate the intensity of the symptoms of anxiety and depression. They were developed by Beck $^{(24)}$ and their Portuguese version was validated by Cunha and Gomes-Oliveira),(25,26). Both have 21 categories, in BAl each of them have 4 alternatives that should be evaluated by the subject on a four point scale: 1- "not at all"; 2- "mildly"; 3- "moderately"; 4- "severely". The scores of anxious symptoms are as follows: 0-10: minimal anxiety; 11-19: mild anxiety; 20-30: moderate anxiety; 31-63: severe anxiety. Whereas the BDI presents four responses in each category with a score varying from 0 to 3 , in which 0 means the absence and 3 the presence of greater intensity of symptoms, among which the subject chooses the most applicable to him/her to describe how he/she felt in the past two weeks, including the day of application of the questionnaire ${ }^{(24)}$. These items relate to increasing levels of severity of depression, and the total score is a result of the sum of the individual items, being able to reach a maximum of 63 points. The interpretation of scores is performed as follows: scores from 0 to 11 minimal or none depression; 12 to 19 mild depression; 20 to 35 moderate depression; 36 to 53 severe depression.

\section{QUALITY OF LIFE QUESTIONNAIRE - EORTHC QLQ-C30}

To assess the QOL of cancer patients, it was authorized by the authors the use of the European Organization for Research and Treatment of Cancer Core Quality of Life Questionnaire EORTHC QLQ-C30. It was developed initially in 1987 by the European Organization for Research and Treatment of Cancer Core, and in 1993 was published the EORTC QLQ-C30 version, which has been improved and is now in its third version, 
$2000^{(27)}$. Contains 30 items related to 16 domains that form 4 scales: global health and quality of life: 1 domain with 2 items; symptoms: 9 domains with 12 items; functional: 5 domains with 15 items; and social: 1 domain with 1 item. The score is obtained according to the patient's response, in which the "not at all" option indicates a point, "a little" two points, "quite a bit" three points or "very much" four points. The scores on the questionnaire range from 0 to 100, in which higher values on functional scales reveal better health status and on symptoms scales reveals greater problems, worsening the quality of life. This questionnaire was translated and validated in Brazil(28) and has been shown to be applicable for the evaluated population.

\section{QUALITY CONTROL}

The researchers responsible for data acquisition in this study will receive specific training to ensure data quality. Periodic external monitoring will be performed to verify the correct application of the methodology for acquiring information and conducting different tests.

\section{STATISTICAL ANALYSIS}

First, a pilot study will be carried out in order to determine the calculation of the sample size. The categorical data will be described as absolute number and percentage of the total. The numerical data will be presented as mean and standard deviation in the case of variables with normal distribution, and median and interquartile range for those with asymmetric distribution. According to the sample stratification, will be used the Student's $t$ test for paired samples. The Kolmogorov-Smirnov normality test will be performed in order to determine the presence or not of normality of the data. For the comparisons between variables, for the quantitative variables, will be used the Student's $t$ test or the non-parametric Mann-Whitney test. When the variables were qualitative, the Chi-square or Fisher's exact test will be used as appropriate. Correlations between continuous variables will be performed with the Pearson correlation coefficient or Spearman's rank correlation coefficient. For statistical treatment, will be used the Statistical Package for Social Sciences, SPSS $18.0^{\circledR}$ (IBM.com, Chicago, IL, USA). The level of statistical significance will be set at $5 \%$ for all tests $(p<0.05)$.

\section{DISCUSSION}

Sleep disorders are increasingly recognized as an important cause of morbidity and mortality. The poor quality of sleep is among the most frequent problems which cancer patients have during the treatment. This study will aim to verify the prevalence of sleep disorders in cancer patients attended at a reference hospital in the Center-West of Minas Gerais, Brazil. Currently, subjective instruments are used to evaluate the sleep pattern in cancer patients, since there is little information investigating the sleep disorders in these subjects. Recognizing these changes is fundamental as the symptoms can negatively affect the quality of life and daytime performance.

\section{ACKNOWLEDGEMENTS}

LTP and CAS receives a grant from the Fundação de Amparo à Pesquisa do Estado de Minas Gerais (local acronym FAPEMIG - PIBIC/UEMG/FAPEMIG). WMS and LFM receives grant from the Programa Institucional de Apoio à Pesquisa da UEMG (local acronym PAPq/UEMG), and SCV receives grant from the Programa Institucional de Apoio à Extensão da UEMG (local acronym PAEx/UEMG).

\section{AUTHOR'S CONTRIBUTIONS}

All authors contributed to the conception and design the study. NSFJ and CSD provided idea for the research or article, created the hypothesis, wrote the original proposal and are the guarantor of the paper. NSFJ, CSD, LTP, WMS and CAS significantly contributed to the writing of this paper. SCV, LFM, JA, RLB, EFCF and CMP were involved in revising the manuscript critically. This protocol paper was written by NSFJ, LTP and WMS with assistance of all co-authors. All authors read and approved the final manuscript

\section{CONFLICTS OF INTEREST}

The authors declare that there was no conflict of interests.

\section{AUTHORS DETAILS}

'Undergraduate student with PIBIC/UEMG/FAPEMIG scholarshipof University of the State of Minas Gerais - Divinópolis Unit (UEMG), Divinópolis, Minas Gerais, Brazil.

2Undergraduate student with PAPq/UEMG scholarshipof University of the State of Minas Gerais - Divinópolis Unit (UEMG), Divinópolis, Minas Gerais, Brazil.

${ }^{3}$ Undergraduate student with PAEX/UEMG scholarshipof University of the State of Minas Gerais - Divinópolis Unit (UEMG), Divinópolis, Minas Gerais, Brazil. ${ }^{4}$ Undergraduate student of University of the State of Minas Gerais - Divinópolis Unit (UEMG), Divinópolis, Minas Gerais, Brazil.

${ }^{5} \mathrm{PhD}$ student in Cellular Biology, Morphology Departament, Institute of Biological Sciences of Federal University of Minas Gerais (UFMG).

${ }^{6}$ Physiotherapist, Specialist in Oncology and Functional Dermato - Association Against Cancer in the Center West of Minas, Divinópolis, Minas Gerais, Brazil.

\section{REFERENCES}

1. Dement WC. A personal history of sleep disorders medicine. J. Clin. Neurophysiol. 1990;7(1):17-47.

2. Luyster FS, Strollo PJ, Zee PC, Walsh JK. Sleep: a health imperative. Sleep. 2012;35(6):727-734

3. Morais LC, Zanuto EAC, Queiroz DC, Araújo MYC, Rocha APR, Codogno JS.Associação entre distúrbios do sono e doenças crônicas em pacientes do sistema único de saúde. J. Phys. Educ. 2017;28(1):2844.

4. AASM. American Academy of Sleep Medicine. The AASM Manual for the scoring of sleep and associated events. Rules, terminology and technical especifications. 2007.

5. Sateia MJ. International Classification of Sleep Disorders - Third Edition. Highlights and Modifications. Chest. 2014;146(5):1387-1394.

6. Campos-Rodriguez F, Martinez-Garcia MA, Martinez M, Duran-Cantolla J, Peña MDL, Masdeu MJ, Gonzalez M, Campo F, Gallego I, Marin JM, Barbe F, Montserrat JM. Association between obstructive sleep apnea and cancer incidence in a large multicenter Spanish cohort. American journal of respiratory and critical care medicine. 2013;187(1):99-105.

7. Kryger MH, Roth T, Dement WC. Principles and Practice of Sleep Medicine: Elsevier Saunders. 2005;4:13-23. 
8. Gondim LMA, Matumoto LM, Melo Júnior MACD, Bittencourt S, Ribeiro UJ. Estudo comparativo da história clínica e da polissonografia na síndrome da apnéia/hipopnéia obstrutiva do sono. Rev Bras Otorrinolaringol. 2007;73(6): 733-7.

9. Kaplow R. Sleep deprivation and psychosocial impact in acutely ill cancer patients. Crit Care Nurs Clin North Am. 2005;17(3):225-237.

10. INCA. Estimativa 2018: Incidência de Câncer no Brasil/ Instituto Nacional de Câncer José de Alencar Gomes da Silva. Coordenação de prevenção e vigilância - Rio de Janeiro: INCA, 2017.

11. Fleming L, Gillespie S, Espie C. The development and impact of insomnia on cancer survivors: A qualitative analysis. Psycho-Oncology. 2010 Sep;19(9):991-996.

12. Vachani C. Insomnia in the patient whit cancer. Oncolink - Abramson Cancer Center of the University of Pensylvania. 2007; 1.

13. Engstrom CA, Strohl RA, Rose L, Lewandowski L, Stefanek ME. Sleep alterations in cancer patients. Cancer Nurs. 1999;22(2):143-148.

14. Rafihi-ferreira R, Pires MLN, Soares MRZ. Sono, qualidade de vida e depressão em mulheres no pós-tratamento de câncer de mama. Psicol. Reflex. Crit. 2012;25(3):506-513.

15. Erik von E, Douglas GA, Stuart JP, Peter CG, Jan PV. Strengthening the Reporting of Observational Studies in Epidemiology (STROBE) statement: guidelines for reporting observational studies. BMJ. 2007;335(7624):806808.

16. Zen V., Fuchs FD, Wainstein MV, Gonçalves SC, Biavatti K, Riedner CE, Fuchs SC. Neck circumference and central obesity are independent predictors of coronary artery disease in patients undergoing coronary angiography. American journal of cardiovascular disease. 2012; 2(4), 323.

17. WHO. Obesity: preventing and managing the global epidemic. Report of a WHO Consultation. World Health Organization, Geneva. 2000;894:1-253.

18. Buysse DJ, Reynolds CF, Monk TH, BermanSR, Kupfer DJ. The Pittsburgh Sleep Quality Index: a new instrument for psychiatric practice and research. Psychiatr Res. 1989;28(2):193-213.
19. Bertolazi AN, Fagondes SC, Holf LS.Validation of the Brazilian portuguese language of the Pittsburgh Sleep Quality Index. Sleep Med. 2011;12(1):70-5.

20. Chiu HY, Chang LW, Hsieh YJ, Tsai PS. A meta-analysis of diagnostic accuracy of three screening tools for insomnia. Journal of Psychosomatic. 2016;87:85-92

21. Castro LS, Poyares D, Leger D, Bittencourt L, Tufik S. Objective prevalence of insomnia in the Sao Paulo, Brazil epidemiologic sleep study. Ann Neurol. 2013;74 (4):537-546.

22. Slater G, Steier J. Excessive daytime sleepiness in sleep disorders. J Thorac Dis. $2012 ; 4(6): 608$

23. Netzer NC, Stoohs RA, Netzer CM, Clark K, Strohj KP. Using the Berlin Questionnaire to identify patients at risk for the sleep apnea syndrome. Ann Intern Med. 1999;131(7):485-1999.

24. Beck AT, Epstein N, Brown G, Steer R. An inventory for measuring clinical anxiety: psychometric properties. American Psychological Association. 1988;56(6):893-897.

25. Cunha JA. Manual da versão em português das Escalas Beck. São Paulo: Casa do Psicólogo; 2001.

26. Gomes-Oliveira MH, Gorenstein C, Lotufo Neto F, Andrade LH, Wang YP.Validation of the Brazilian Portuguese version of the Beck Depression Inventory-II in a community sample. Rev Bras Psiquiatr. 2012;34(4):389394.

27. Peter F, Aaronson NK, Bjordal K, Groenvold M, Curran D, Bottomley A. EORTC QLQ-C30 Scoring Manual (3rd edition). On behalf of the EORTC Quality of Life Group. Brussels: EORTC. 2001.

28. Silva FA. Validação e reprodutibilidade de questionários de qualidade de vida específicos para câncer de mama. Dissertação de Mestrado. São Paulo: Fundação Antônio Prudente; 2008. 